\title{
Non-Newtonian Visco-elastic Heat Transfer Flow Past a Stretching Sheet with Convective Boundary Condition
}

\author{
P H Veena, D Vinuta, V K Pravin \\ Dept. of Mathematics, Smt. V.G. College for Women, Gulbarga, Karnataka, India \\ Dept. of Mathematics, Gulbarga University, Gulbarga, Karnataka, India \\ Dept. of Mech. Engg., P.D.A. College of Engg., Gulbarga, Karnataka, India
}

\begin{abstract}
In this paper two dimensional flow of a viscoelastic fluid due to stretching surface is considered. Flow analysis is carried out by using closed form solution of fourth order differential equation of motion of viscoelastic fluid. Further (Walters' liquid B' model) heat transfer analysis is carried out using convective surface condition. The governing equations of flow and heat transfer are non-linear partial differential equations which are unable to solve analytically hence are solved using Runge-Kutta Numerical Method with efficient shooting technique. The flow and heat transfer characteristics are studied through plots drawn. Numerical values of Wall temperature are calculated and presented in the table and compared with earlier published results which are in good agreement
\end{abstract}

Key Words: Visco-elastic (Walters' liquid B') fluid, stretching sheet, convective boundary condition, heat transfer, flow analysis

\section{Nomenclature \\ b stretching rate \\ $x \quad$ horizontal coordinate \\ $y \quad$ vertical coordinate \\ $u \quad$ horizontal velocity component \\ $v \quad$ vertical velocity component \\ $T$ temperature \\ $c_{p} \quad$ specific heat \\ $f$ dimensionless stream function \\ Pr Prandtl number \\ $l \quad$ Characteristic length \\ $\mathrm{k}_{1} \quad$ viscoelastic parameter \\ $k_{0} \quad$ the co-efficient of elasticity, \\ $T_{f} \quad$ the temperature of the sheet. \\ $B_{i} \quad$ Biot number \\ differentiation with respect to $\eta$}

\section{Greek symbols}

$\eta \quad$ similarity variable

$\theta \quad$ dimensionless temperature

$k \quad$ thermal conductivity

$\mu \quad$ viscosity

$v \quad$ kinematic viscosity

$\rho$ density

$\alpha \quad$ root value

\section{Subscripts}

$w \quad$ properties at the plate

$\infty \quad$ free stream condition

\section{INTRODUCTION}

In reality most of the liquids are nonNewtonian in nature and are used abundantly in many engineering applications, such as plastic film manufacture, artificial fibers manufacture, aerodynamic extrusion of plastic sheets, cooling of metallic sheets in a cooling bath on process, in Geothermal reservoirs and in petroleum industries. Hence the study of viscoelastic non-Newtonian fluid flow and heat transfer phenomena is much more important as considered to the study of Newtonian fluids. Many researchers worked on the flow and heat transfer problems of viscoelastic fluids. Some of them are discussed below.

As we study the literature, Sakiadis [1] was a first researcher among all other investigators to study the phenomena considering boundary layer flow of viscous fluid over moving rigid surfaces. It is more appropriate to consider non-Newtonian behavior of all those fluids in the analysis of boundary layer flow and heat transfer characteristics as most of the fluids such as plastic films, and artificial fibers in industrial applications are strictly Newtonian. But these were restricted to flow and heat transfer in non-porous media. In recent years a great deal of interest has focused on the rheological effects of non-Newtonian flow through porous media.

Rajagopal et.al [2] studied the flow of viscoelastic fluid over a stretching sheet. Siddappa and Abel [3] studied the flow analysis and heat transfer characteristics of viscoelastic fluid due to stretching plate in presence of suction and 
injection. Further the same authors Siddappa and Abel [4] studied the visoelastic fluid flow analysis due to stretching sheet. They obtained the analytical solution for the problem. Dandapat and Gupta [5] studied the flow and heat transfer charecteristics in a visco-elastic fluid over a stretching sheet. Lawrence and Rao [6] investigated the heat transfer analysis in the flow of a viscoelastic fluid over a stretching sheet. Cortell [7] studied the similarity solutions of the flow and heat transfer analysis of visco-elastic fluid due to a stretching sheet. Sonth et.al [8] analyzed the heat and mass transfer effects in viscoelastic fluid flow over on accelerating surface with effect of heat source/sink and viscous dissipation. Khan and Sanjayanand [9] investigated the visco-elastic fluid flow and heat transfer analysis using an exponential stretching sheet. Bhattacharya et.al [10] studied the heat transfer of a visco-elastic fluid over a stretching surface.

Nataraja et.al [11] investigated the nonSimilar solutions for flow and heat transfer problem considering a viscoelastic fluid over a stretching sheet. Abel et.al [12] investigated the flow and Heat transfer characteristics in a viscoelastic boundary layer flow over a stretching sheet with viscous dissipation and non-uniform heat source. They obtained an analytical solution for the problem in terms of Kummer's function. Aziz [13] has given a similarity solution for laminar thermal boundary layer over flat plate with a convective boundary condition for the viscous fluid. Makinde and Aziz [14] investigated the mixed convection on MHD flow of viscous fluid from a vertical plate embedded in a porous medium with a convective boundary condition is used to study the heat transfer analysis.

$$
\begin{aligned}
& \frac{\partial u}{\partial x}+\frac{\partial v}{\partial y}=0 \\
& \text { (1) } u \frac{\partial u}{\partial x}+v \frac{\partial v}{\partial y}=v \frac{\partial^{2} u}{\partial y^{2}}-k_{0}\left\{u \frac{\partial^{3} u}{\partial x \partial y^{2}}+v \frac{\partial^{3} u}{\partial y^{3}}+\frac{\partial u}{\partial x} \frac{\partial^{2} u}{\partial y^{2}}-\frac{\partial u}{\partial y} \frac{\partial^{2} u}{\partial x \partial y}\right\} \\
& \rho c_{p}\left(u \frac{\partial T}{\partial x}+v \frac{\partial T}{\partial y}\right)=k \frac{\partial^{2} T}{\partial y^{2}},
\end{aligned}
$$

Here in above equations, $\rho$ is the density, $\mathrm{T}$ is the temperature, $v$ is the kinematic viscosity, $k_{0}$ is the co-efficient of elasticity, $k$ is coefficient of thermal conductivity, $c_{p}$ is specific heat at constant pressure, The other quantities have their usual meaning. It is to be emphasized here that Eq.
Makinde and Olanrewaju [15] investigated the Buoyancy effects on the thermal boundary layer over flow due to a vertical plate with a convective surface boundary condition for heat transfer analysis. Yao and Fang [16] investigated the heat transfer analysis of a generalized stretching /shrinking wall problem with convective boundary conditions. Makinde and Aziz [17] studied boundary layer flow of a nano-fluid past a stretching sheet with a convective boundary condition.

On observing above there are studies of heat transfer analysis of viscous fluids with convective heating conditions, but there are no studies in heat transfer analysis of viscoelastic fluids with convective heating condition as it has more practical application in the field of engineering and industries. Hence in the present paper the investigation of flow and heat transfer analysis of viscoelastic fluid with convective heating boundary condition is studied.

\section{MATHEMATICAL FORMULATION}

Here it is considered the two-dimensional laminar boundary layer flow of an incompressible, visco-elastic fluid (Walters' liquid B' model) due to a stretching sheet as it has created due to two equal and opposite forces applied along the $\mathrm{x}$-axis, so that the sheet is stretched, keeping the origin fixed. Under the boundary layer approximations and the assumptions that the contribution due to the normal stress is of the same order of magnitude as the shear stress - the basic boundary layer equations governing the flow of Walters' Liquid B', heat transfer in presence of non-uniform heat generation can be written as: 


$$
\begin{aligned}
& \left.\begin{array}{cc}
u=b x, & v=0, \\
-k \frac{\partial T}{\partial y}=h_{f}\left(T_{f}-T\right)
\end{array}\right\} \\
& \left.u \rightarrow 0, \frac{\partial u}{\partial y} \rightarrow 0, T \rightarrow T_{\infty}\right\} \quad \text { as }
\end{aligned}
$$

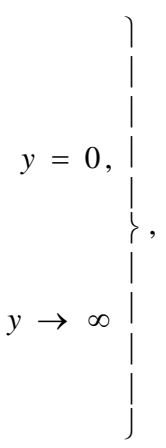

Here $\mathrm{u}$ and $\mathrm{v}$ are the velocity components along $\mathrm{x}$ - and y-directions respectively, where $T_{\infty}$ is the temperature of the fluid far away from the sheet (temperature of ambient cold fluid) $\mathrm{T}$ is the uniform temperature on the top surface of the plate. Hence we have $T_{f}>T>T_{\infty}$.

\section{FLOW ANALYSIS}

Now introducing the similarity transformations in the form,

$u=b x f_{\eta}(\eta), v=-\sqrt{b v} f(\eta)$

where $\eta=\sqrt{\frac{b}{v}} y$ is dimensionless normal

distance

Eq.(1) is identically satisfied with these change of variables and Eq.(2) is transformed to:

$$
f_{\eta}{ }^{2}-f f_{\eta \eta}=f_{\eta \eta \eta}-k_{1}\left\{2 f_{\eta}-f f_{\eta \eta \eta \eta}-f_{\eta \eta}{ }^{2}\right\}
$$

where

$k_{1}=\frac{k_{0} b}{v}$,

the subscript $\eta$ denotes the differentiation with respect to $\eta, k_{1}$ is the non-dimensional viscoelastic parameter. The governing boundary conditions on velocity Eq.(4) take the following form as:

$$
f=0, \quad f_{\eta}=1 \quad \text { at } \quad \eta=0,
$$

(8)

$$
f_{\eta} \rightarrow 0, \quad f_{\eta \eta} \rightarrow 0 \quad \text { as } \quad \eta \rightarrow \infty
$$

The solution of Eq.(6) corresponding to the boundary conditions (8) is obtained as

$$
f(\eta)=\frac{1-e^{-\alpha \eta}}{\alpha},
$$

where

$$
\alpha=\sqrt{\frac{1}{1-k_{1}}} .
$$

The velocity components u \& v become

$$
u=b x e^{-\alpha \eta}, v=-\sqrt{b v}\left(\frac{1-e^{-\alpha \eta}}{\alpha}\right)
$$

where $b>0$ and $\alpha$ is given by Eq. (10).

\section{SKIN FRICTION}

The local skin friction coefficient or frictional drag coefficient is given by

$$
C_{f}=\frac{\tau_{w}}{\mu b x \sqrt{\frac{b}{v}}}=\alpha,
$$

where $\tau_{w}=-\mu\left(\frac{\partial u}{\partial y}\right)_{y=0}=\mu b x \alpha \sqrt{\frac{b}{v}}$, is the wall shearing stress on the surface of the stretching sheet, $\mu$ is the dynamic viscosity of fluid and $\alpha$ is given by Eq.(12).

\section{HEAT TRANSFER ANALYSIS}

In this section the convective boundary condition with constant surface temperature for the heating process is considered. To solve heat equation (3) Defining the non-dimensional temperature $\theta(\eta)$ as:

$\theta(\eta)=\frac{T-T_{\infty}}{T_{f}-T_{\infty}}$.

where $T_{f}$ the temperature of the sheet.

Using Eqn. (13), Eqs. (3) and (4) can be converted to

$\theta^{\prime \prime}+\operatorname{Pr} f \theta^{\prime}=0$, 
where

$$
\begin{aligned}
& \operatorname{Pr}=\frac{\mu C_{p}}{k} \text { is the Prandtl number. } \\
& B_{i}=\frac{h}{k} \sqrt{\frac{v}{a}} \text { is the thermal Biot number }
\end{aligned}
$$

Here Biot number $B_{i}$ is the dimensionless parameter and it plays the fundamental role in conduction problems that involves surface convection effects. Biot number parameter provides the measure of temperature drop in the solid relative to the temperature difference between the surface and fluid.

For $\mathrm{Bi}<<1$ shows the resistance to conduction within the solid is much less than the resistance to the convection across the fluid boundary layer, hence assumption of uniform temperature is reasonable. Whereas $\mathrm{Bi}>>1$ says the temperature difference across the solid is much larger than that between surface and fluid.

\section{NUMERICAL SOLUTION}

In this section we are explaining about the method of Numerical Solution used to solve the boundary value problems of considered study. The Governing equations of motion and heat transfer are highly non-linear so it is very difficult to solve, hence the partial differential equations are converted into ordinary differential equations by means of similarity transformation. The exponential exact solution of momentum equation is obtained and is used to find the solution of energy equation (14) with respect to the boundary conditions (15). The Boundary value problem considered is converted into initial value problem then by using Newton-Rapson method, the missing condition is found and then using Runge-Kutta integration scheme the solution of temperature boundary value problem is obtained. The step size is chosen as 0.001 and the accuracy of result is maintained upto 10-6.

\section{RESULTS AND DISCUSSION}

After obtaining the solution of boundary value problem, various plots of flow, velocity and temperature are plotted to analyze the effect of various governing parameters.

Fig.1. is the representation of physical sketch of considered problem. It shows how the stretching sheet problem is constructed.

Fig.2. Is plotted for the flow profiles as well as the velocity profiles for different values of viscoelastic parameter $\mathrm{k} 1$. Which show that, both the profiles decrease with increase in the parametric values of viscoelastic parameter which happens throughout the boundary layer which is obvious.

Fig.3 . is Plotted for the temperature profile which shows the effect of viscoelastic parameter. On observing the plot one can see that the temperature is increasing with increase in the paramentric value of viscoelastic parameter. This is due to the fact that an increase of viscoelastic normal stress giverise to thickinning of the thermal boundary layer.

Fig. 4 shows that, the temperature profile decreses with an increase in the parametric value of Prandtl number, which shows that the viscoelastic boundary layer is thicker than the thermal boundary layer.

Fig. 5 shows the effect of Biot number parameter on the temperature profile. On observing the graph it depicts the fact that temperature is increases with increase in the parametric value of Biot number as the stronger convection results in higher surface temperatures, which causes the thermal effect to penetrate strongly deeper into the fluid.

\section{CONCLUSIONS}

- Analytical Solutions for flow and numerical solution of convective heat transfer problem are obtained.

- The effects viscoelastic parameter is to decrease the flow and velocity, which was quite opposite on temperature.

- The thermal boundary layer thickness decreases with increasing Prandtl number in convective heat transfer phenomenon

- When viscoelastic parameter $\mathrm{k}_{1}$ tends to zero, results are reduced to the Newtonian case.

\section{REFERENCES}

[1]. Sakiadis BC; Boundary layer behavior on continuous solid surfaces AICHEJ 1961:7:26-8.

[2]. Rajagopal KR, Na TY, Gupta AS; Flow of a viscoelastic fluid over a stretching sheet Rheol Acta 1984;23:213-5.

[3]. Siddappa B, Subhas Abel; Visco-elastic boundary layer flow past a stretching plate with suction and heat transfer. Rheol Acta, 1985(23)815-16.

[4]. Siddappa B, Subhas Abel; Non-Newtonian flow past a stretching plate, ZAMP, 1985(36) 890-892.

[5]. Dandapat BS, Gupta AS; Flow and Heat transfer in a visco-elastic fluid over a stretching sheet. Int .J.Non-linear Mech.1989;24(93);21 5-9 
[6]. Lawrence PS, Rao BN; Heat transfer in the flow of a visco-elastic fluid over a stretching sheet. Acta Mech.1992 ;(93); 53-61.

[7]. Cortell R; Similarity solutions for flow and heat transfer of visco-elastic fluid over a stretching sheet. Int .J.Non-linear Mech 1994 29(2):155-61.

[8]. Sonth RM, Khan SK, Abel MS, Prasad KV; Heat and mass transfer in viscoelastic fluid flow over on accelerating surface with heat source/sink and viscous dissipation. Heat Mass Transfer 2002:38:213-20.

[9]. Khan SK, Sanjayanand E; Visco-elastic boundary layer flow and heat transfer over an exponential stretching sheet. Int .J.Heat Mass Transfer 2005.48.1534-42.

[10]. Bhattacharya.S, Pal.A, Gupta.A.S; Heat transfer in the flow of a visco-elastic fluid over a stretching surface Heat Mass Transfer 34(1998)41-45.

[11]. Nataraja H.R., Sharma.M.S., Nageshwar Rao B; Non-Similar solutions for flow and heat transfer in a visco-elastic fluid over a stretching sheet. Int .J. non-linear mech.33 (1998)357-361.

[12]. Abel, Siddheshwar.M.S, PG, Mahantesh M.Nandeppanavar; Heat transfer in a viscoelastic boundary layer flow over a stretching sheet with viscous dissipation and non-uniform heat source. Int. J. Heat Mass Transfer 2007:50:960-6.

[13]. Aziz.A; A similarity solution for laminar thermal boundary layer over flat plate with a convective boundary condition. Commun. Non-linear Sci, Numer. Simulat 14(2009) 1064-1068.

[14]. Makinde.O.D, Aziz.A; MHD mixed convection from a vertical plate embedded in a porous medium with a convective boundary conditions. Int.J.Ther.Sci.49 (2010)1813-1820.

[15]. Makinde.O.D, Olanrewaju.P.O; Buoyancy effects on thermal boundary layer over a vertical plate with a convective surface boundary condition. Trans.ASME-J.Fluid Eng.132 (2010)044502(1-4).

[16]. Yao.S, Fang.T; Heat transfer of a generalized stretching /shrinking wall problem with convective boundary conditions. Commun.Non-linear Sci Numer, simulate.16 (2011)752-760.

[17]. Makinde.O.D., Aziz.A; Boundary layer flow of a nanofluid past a stretching sheet with a convective boundary condition. Int J.Therm.Sci.50 (2011)1326-1332.

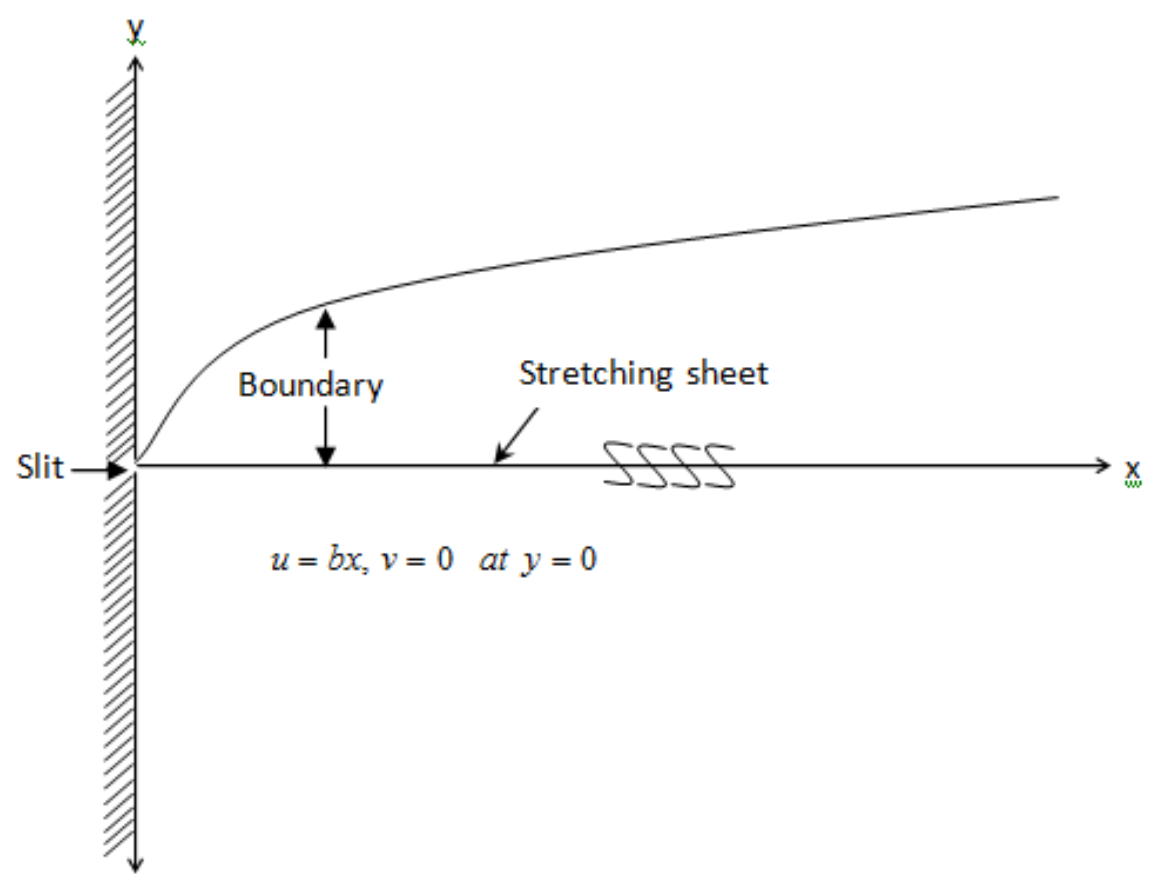

Fig. 1. Schematic diagram of linear stretching sheet 


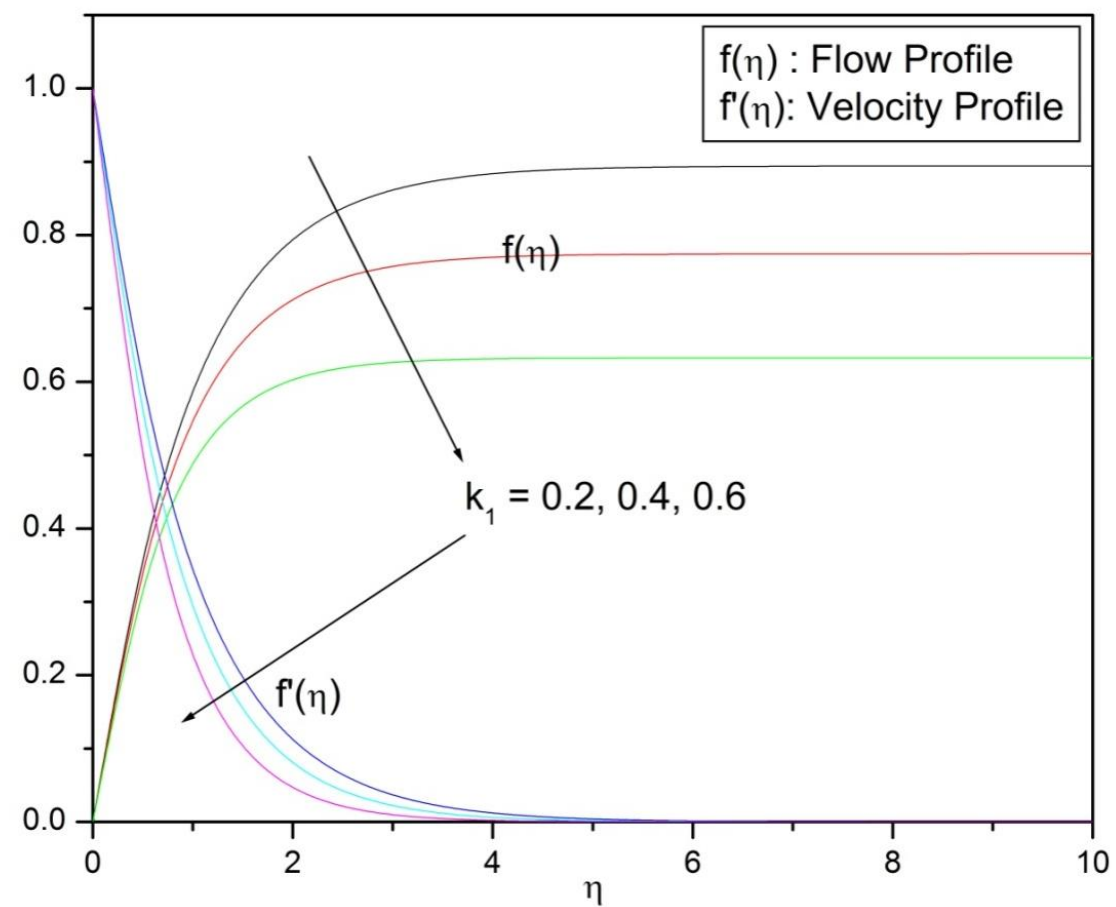

Fig.2. Flow and Velocity profile for different values of viscoelastic parameter



Fig.3. Temperature profiles for different values of visco-elastic parameter 


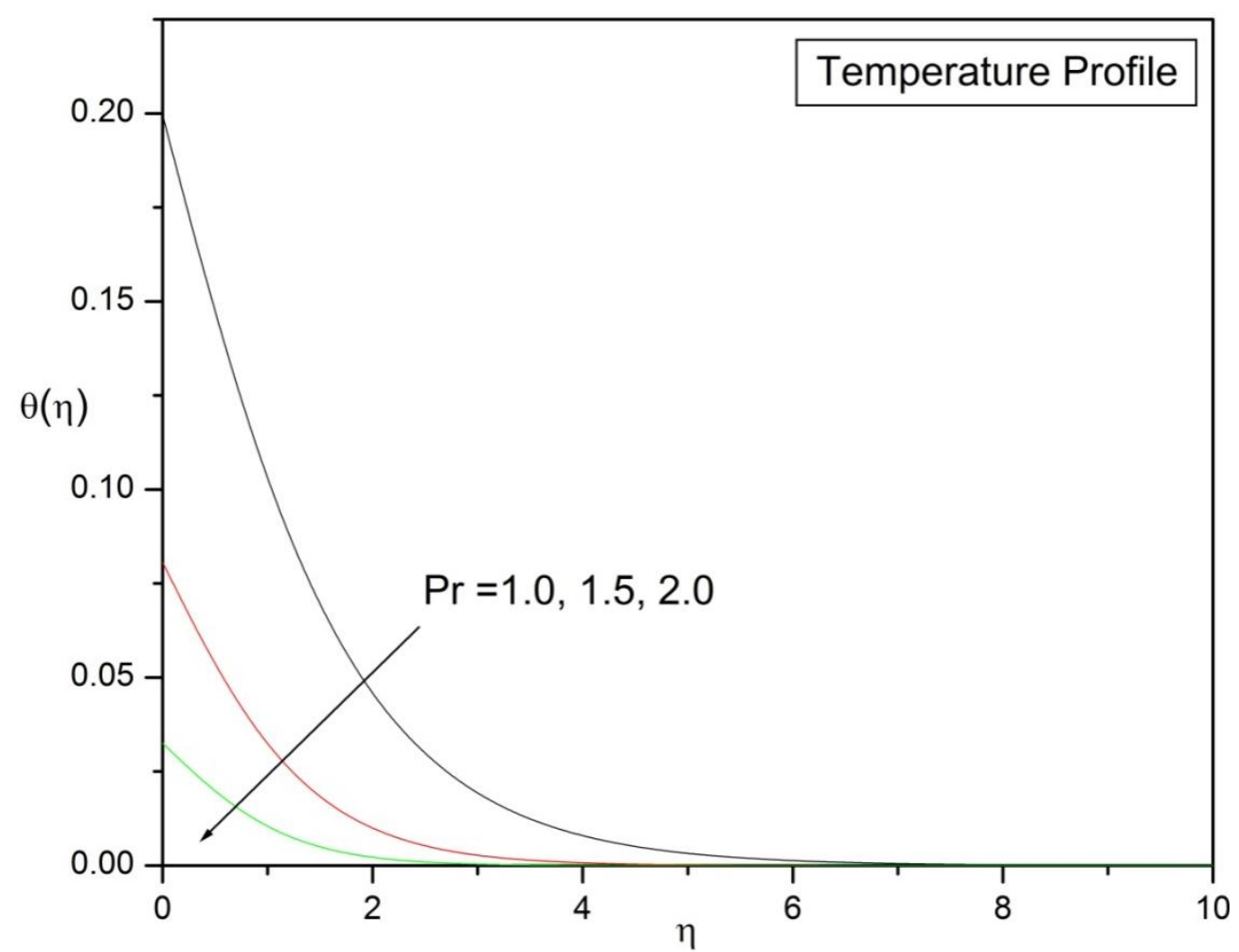

Fig..4. Temperature profiles for different values of Prandtl number

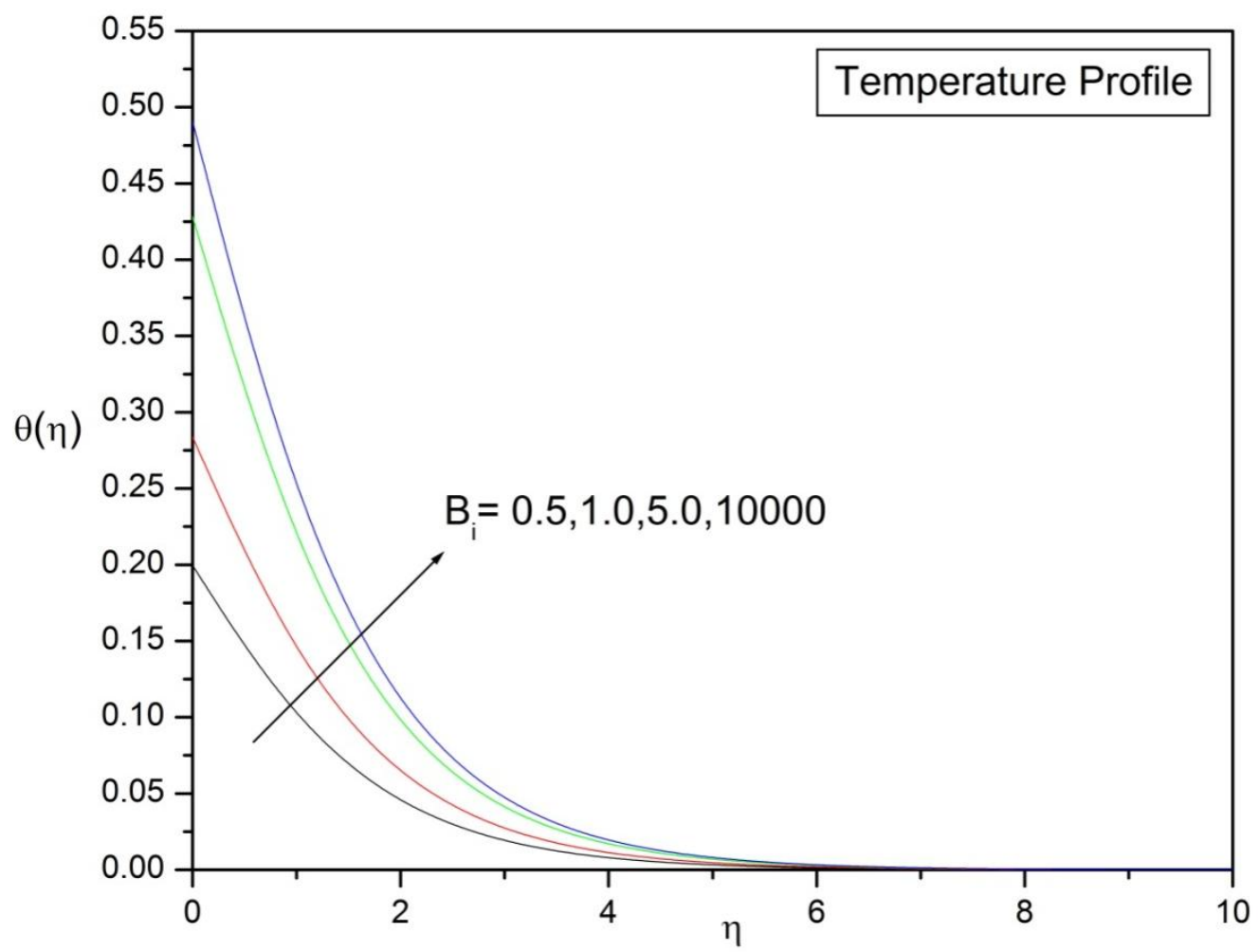

Fig.5. Temperature profiles for different values of Biot number $B_{i}$ 